\title{
LII. On reducing the lunar distance
}

\section{Henry Meikle Esq.}

To cite this article: Henry Meikle Esq. (1822) LII. On reducing the lunar distance, Philosophical Magazine Series 1, 60:294, 271-279, DOI: 10.1080/14786442208652839

To link to this article: http://dx.doi.org/10.1080/14786442208652839

$$
\text { 曲 Published online: } 29 \text { Jul } 2009 .
$$

Submit your article to this journal 전

Q View related articles 두 
manner Mr. Russell has supposed; and I leave it to the philosophers to make their choice between the two hypotheses.

I am, gentlemen,

Your most obedient servant,

Bath, August 2, 1822.

Walter Forman.

P.S.-I cannot take leave of this subject wichout pointing out to the notice of your readers a most extraordinary feature in Mr. Russell's mode of arguing (if I may be allowed that expression), which is certainly unique in the annals of philosophy. Mr. Russell denies my hypothesis, and proposes another; but he does not think it necessary either to prove that his own is true, or that mine is false. He asserts that the tides are raised "by the superior gravity of those waters that constitute the ebb;" but where are his proofs? He does not explain in what way the downward pressure of the waters on one part of the globe can cause the waters on another part to rise up; and yet he expects me to combat a position, when it is morally impossible that I can understand what he means.

As however he has thought proper to throw the burden upon my shoulders, I am ready to undertake it; because, though I cannot be expected to combat an argument before it is put, it is easy to show that his hypothesis cannot possibly be true. Mr. Russell, I suppose, is so much of a philosopher as to know that, whenever it is low waicr in any place (let him choose where he will), the tides are rising on one side of it and ebbing on the other; and, if the "superior gravity" of the water in this place cannot prevent the waters from ebbing on one side, it is not very philosophical to suppose that it cau lift the waters on the other.

\section{On reducing the LunarDistance. By Henry MeIKLE, Esq.*}

\section{To the Editors of the Philosophical Magazine and Journal.}

Gentrenen,- IN my communication which you had the goodness to insert in the Philosophical Magazine for September last, a method was proposed for constructing a general plate for reducing the lunar distance; and its leading feature, so far as relates to parallax, was shown to consist in the arrangement of three parallel straight lines in such a manner that when two of them served for the altitudes, the effect of parallax on the distance might by help of a ruler be read off from the third. The same general principle, it was also remarked, admitted of several forms of construction; and since

* Communicated by the Author, 
that time I have made some comparison among these different forms, with the view of ascertaining which of them is on the whole likely to be the most convenient in practice. This has at length inclined me rather to prefer the one in which the line for correction has only one position, whilst the lines of altitude change their places for each degree of distance. In it the parts of the various lines are better proportioned to suit the several distances, it is more applicable to the solution of other problems, and it also admits of a convenient mode of reading off the correction in terms of any horizontal parallax. I shall therefore describe more particularly that construction in which the same line of correction answers alike for every distance, and in which there is only one line for the moon's altitude, whilst the star's altitude takes a different line for each degree of distance.

Let $\mathrm{MN}$ as formerly be a line of sines whose zero is at $M$, and parallel to it draw IT equal the horizontal parallax; divide $I$ 'T into a line of sines beginning from $T$, and produce it to contain another equal line of sines ' $T I^{\prime}$, also beginning at ' $T$, but in the opposite direction.

Now to find the position of the line of star's altitude for a given distance: Lay a ruler from the sine of the distance

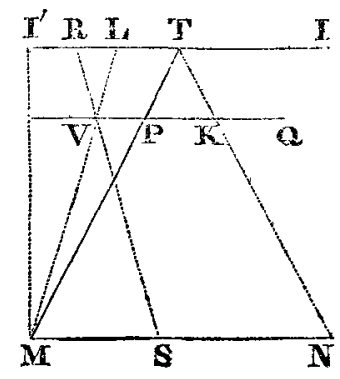
taken on MN to its cosine reckoned on ' $\mathrm{II}^{\prime}$; then through the point $\mathrm{P}$ where the ruler cuts $\mathrm{MT}$ draw $P Q$ parallel to $M N$, and it is the position required.

Next to find the divisions on $P Q$ for the sine of each degree of the star's altitude: Imagine the corresponding distance to be in a vertical circle, and lay a ruler from the sine of the moon's altitude on $M N$ to the cosine of her altitude on $I^{\prime} T$ if the effect of parallax is additive, or if the distance exceed $90^{\circ}$, but on I $T$ when that effect is subtractive and the distance also less than $90^{\circ}$ : the ruler will then cut $\mathrm{PQ}$ in the point that is to be marked with the intended degree of the star's altitude.

This latter precept obviously comprehends the preceding one, and the investigation formerly given is applicable to both; for by this construction, when $\mathrm{N}^{\prime} \mathrm{T}$ ' is joined, it cuts any line of star's altitude $\mathrm{PQ}$ in a point $\mathrm{K}$, such that $\frac{\mathrm{PK}}{\mathrm{PQ}}=\cos d$. So that, retaining the former notation, if $\mathrm{SV} M \mathrm{MV}$ be drawn and produced to meet $I I^{\prime}$ in $R$ and $L$, we have just the same state of things as before. In this arrangement, the lines of star's altitude for distances greater than $90^{\circ}$ evidently lie to the left 
of MT; and the corresponding parts of such a line for any distance above $90^{3}$ are just equal to those belonging to a distance as much under $90^{\circ}$; both being in one straight line, and reckoned from the same point of MT, although in opposite directions. If I'M be joined, it forms the limit of any line of star's altitude that lies to the left of $M^{\top} T$ : since the part that would fall without $I^{\prime} M$ can never come into use; because both $I M$ and $I^{\prime} M$ cut every line of star's altitude in the point which answers to sine of the distance. This form of construction is therefore more compact than the one formerly described.

When the distance is less than $90^{\circ}$, the additive correction lies to the left of $T$, and the subtraction to the right; but if the distance is not less than $90^{\circ}$, the correction is always subtractive. This correction for parallax may be obtained nearly as directed in the former paper, by laying a ruler from the moon's altitude on $\mathrm{MN}$ to that of the star on the line belonging to the distance: the segment of $I^{\prime} I^{\prime}$ intercepted between the ruler and $T$ will then be the correction in terms of $I T$.

If $\mathrm{I} T=60^{\circ}$, the correction may easily be reduced by practice to suit any other horizontal parallax, especially since the number 60 contains such a variety of divisors, and is besides the radix of the sexagesimal scale;-a circumstance which renders it more convenient for the purpose than any other number.

But perhaps the following would be an easier though less accurate mode of reading off the correction at first in terms of any horizontal parallax:-Draw as many straight lines, all equal and parallel to $I I^{\prime}$, as there are horizontal parallexes differing by one minute: cross these again by another set of parallels drawn pretty close to each other, and divide each of the first set into as many equal parts as there are minutes in the horizontal parallax which it represents. Then having applied the ruler to the altitudes and distance, as already directed, follow one of the cross parallels from the point where the ruler cuts $I^{\prime}$, and it will cross the line representing the given horizontal parallax in the point which indicates the corresponding correction. In this method $I \mathrm{~T}$ should be made either the greatest or least horizontal parallax.

When it is thought necessary, the final effect of parallax may be allowed for in the principal effect as follows: Let the moon's altitude be half corrected for parallax, and therewith find the effect of parallax on distance nearly; then, without removing the ruler, apply half the effect just found to the distance; and by shifting the ruler a very little to suit the distance so corrected, it will give the true effect of parallax. The parallax in altitude may be readily had on I T, \&c. by entering with the moon's zenith distance as an argument.

Vol. 60. No. 294. Oct. 1822.

$\mathrm{M} \mathrm{m}$

The 
The construction that has now been described might assist in giving a solution in round numbers of various problems in spherical trigonometry. Thus, having given the latitude of the place, the declination and altitude of the sun to find the time; in place of the distance, the altitudes of the moon and star, substitute the colatitude, the declination and altitude of the sun respectively; and by attending to the signs of the quantities, we shall have cos. declin. $\times$ cos. horary angle. This again night be freed from cos. declin. by a small separate scale, or by such a method as that proposed for the different horizontal parallaxes.

I have also succeeded in materially improving the construction formerly proposed for showing the effect of refraction; and in addition to other advantages, it does not now require a ruler laid across in using it.

Let $A B$ be a line of sines beginning at $\mathrm{A}$; draw D A C perpendicular to it, and join $\mathrm{BC}$; draw also a set of straight lines parallel to $A B$, and reaching firom $\triangle \mathrm{C}$ to $\mathrm{BC}$ : next lay a ruler from $D$ through each degree of A B, and draw as many straight lines extending from

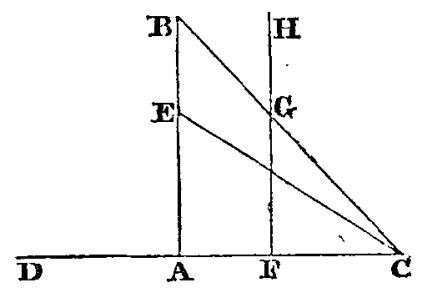

$A B$ to $B C$; these will evidently divide each parallel into a portion of a line of sines, but always to a greater radius, as they are more distant from $D$. From $C$ draw a set of diverging lines to meet $\mathrm{AB}$, and of course dividing all the other parallels in the same ratio as they do AB. Hence if the greater altitude be sought out on $A \mathrm{~B}$, and if from that point $\mathrm{E}$ an oblique line be followed till it meet the less altitude on some other parallel FG, it is plain that every oblique line drawn from $\mathrm{C}$ must also cut $\mathrm{AB}$ and $\mathrm{FG}$ in points which denote two altitudes whose sines are in the same ratio as those of the first two. If therefore $G H$ be the effect of refraction in the first case, it will be so for the same distance whenever the sines of the altitudes are in the same ratio; that is, whenever the less altitude falls on FG. Reckoning then the greater altitude always on A B, it follows that the less of every two altitudes whose sines have the same ratio, must fall on the same parallel; or, that each parallel belongs to a different ratio of the sines of the altitudes. So that a curve may be constructed in the manner formerly directed, of such a nature that when the several parallels are produced to meet it, the part of each produced, or the segment interecpted between B C and the curve may always represent the effect 
effect of refraction on the same distance, whenever the less altitude falls on that parallel.

The use of a linear table constructed in this form for showing the effect of refraction, is therefore very simple: for having found on what parallel the less altitude falls, trace that upward, and its segment intercepted between BC and the curve corresponding to the given distance, is the effect required, which is always additive. This table is not quite half the size of that formerly described, when the corrections in both are on the same scale, and there is sufficient room for inserting it above $\mathrm{MN}$ in the table for parallax. If small altitudes are not to be used, a considerable part of the figure next A C might be omitted, which would still further reduce the size.

It was already remarked that on the same distance, the effect of refraction is nearly the same for any two altitudes whose sines have the same ratio; and to render the above explanation more simple, I only introduced lines of sines. But when the sines are proportionals, their like powers or roots are also proportionals; and therefore the effect of refraction must be nearly the same when the like powers or roots of the sines have the same ratio. Hence, if in place of lines of sines we use their square roots the above construction would be much improved: for by this means the curves being less crowded would meet the parallels much less obliquely when the effect of refraction is great; and thus the different parts of the scale from which the correction is to be read wonld be better proportioned. But I have not yet ascertained what root of the sines would answer best. It was however to effect the same end in some degree, that the lines were to be drawn diverging from $\mathrm{D}$, and not parallel to $\mathrm{A} C$.

I shall now proceed to describe the outline of a very different contrivance for clearing the distance; if this has not every property that the other possessed, it is much more easily constructed, it does not require a ruler laid across, and when of the same size as the former it shows the correction for parallax on twice as great a scale.

In such a spherical triangle as is formed by the distance and the complements of the altitudes, it is in effect demonstrated by almost every writer on spherics, that a perpendicular from the zenith being let fall on the distance, divides it into two segments whose cosines are as the sines of the altitudes; and also, that the tangents of these segments are respectively proportional to the cotangents of the altitudes multiplied by the cosines of the adjacent angles at the base; that is, nearly as the effects of the refractions on the distance. If; therciore, a M m 2 
ready method could be found for dividing the distance into the two proper segments, it would afford the means of obtaining the effect of refraction with considerable facility. This division has sometimes been accomplished by calculation, as in Witchel's method; and can likewise be done by projection; but at present, I wish to show how a very simple diagram may be constructed to serve the purpose, as well as to give the effect of parallax.

Let $A D$ be a square of which the two sides $A B, C D$ are lines of sines whose zeros are at $\mathrm{A}$ and $\mathrm{C}$; the corresponding degrees being joined by straight lines which are of course parallel to each other; and these again are to be divided into sixty equal parts by a set of straight lines parallel to $A B$, which in their turn are divided by the former into lines of sines the same as A B. The lines parallel to $\mathrm{AC}$ are also cut by a curve in such a manner that the segment of each reckoned from DC may represent the refraction corresponding to that altitude, and the same lines are further cut by a circle described from $A$ to $D$ about the centre $C$, so that the segments will always represent the parallax in altitude for a horizontal parallax of $60^{\prime}$ : each of the 60 equal parts being counted one minute of parallax; but to enlarge the scale as much as possible, it is proposed to reckon each of the equal parts to be only $10^{\prime \prime}$ of refraction, and the whole line only ten minutes. From $\mathbf{C}$ draw a set of diverging lines to spread over the whole square; these will obviously cut the vertical lines proportionally, so that if the segments of two vertical lines cut by one obligue line be the sines of the altitudes, the cosines of the segments of the distance will also be segments of the same verticals when both are cut by another oblique line, since these cosines are proportional to the sines of the altitudes.

To find the effect of refraction by this method: Look for the greater altitude in A B, and from that point follow down an oblique line till it meet the less altitude in some other vertical; then follow up at same time the two vertical lines in which the altitudes were found till some oblique line cut them both in such a manner that the sum or difference of the remaining zenith distances may equal the distance; and the sum or difference of the corresponding refractions will be the effect required, which is always additive. There is no ambi-

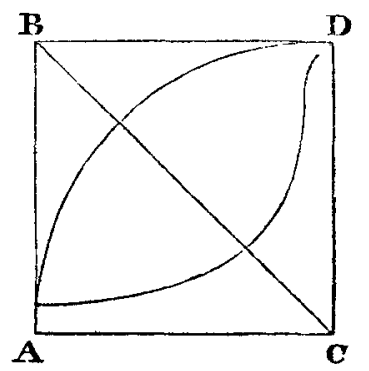
mnity as to whether the stm or difference of the zenith di* stances 
stances is to be taken; because only one of them (the sum or difference) can be equal the distance, unless when one of the remaining zenith distances is nothing; and the sum or difference of the refractions is to be taken according as the sum or difference of the zenith distances was used. The case that requires their difference is easily known in following up the altitudes, since the greater altitude will then seem to go above the top before their sum could be so small as the distance.

Next, to find the effect of parallax: Observe where the horizontal line on which the effect of the moon's refraction was found cuts the circle $A D$; from this point trace dewn an oblique line till it cut the parallel of the moon's altitude, and the segment of this parallel reckoned from $\mathrm{CD}$, is the principal effect of parallax in terms of a horizontal parallax of $60^{\prime}$. For the tangent of segment of distance adjacent to the moon, is equal the cotangent of the moon's altitude multiplied by cosine of angle at the moon; and the quantity we have obtained is obviously equal to tangent of the above segment multiplied by sine moon's altitude; that is, to cosine of angle at the moon multiplied by cosine of her altitude. The above effect is easily reduced to suit any horizontal parallax by the common rule of practice, as was directed in the other method; and the final effect of parallax may be allowed for, when it is deemed necessary, by half correcting the altitudes and clistance, and then repeating the operation to find the true effect of parallax; though this cannot be done quite so conveniently here as in the other method. The correction for parallax is additive, when the difference of the refractions is used and the moon at the same time the higher body. In all other cases it is subtractive.

It may be observed, that in place of a square, A B D C might have been an oblong, and $A D$ the quadrant of an ellipse. Nor is it absolutely necessary that the vertical lines should be lines of sines, or lines of their powers; they might, on the contrary, be divided in any proportion that may be thought to suit better; but then the lines diverging from $\mathbf{C}$ could not always be straight lines, neither could $\mathrm{AD}$ be a circular ellipse. If, however, the rectilineal part of such a diagram as that already described were first constructed, it would be easy from it to draw another in which the vertical limes were divided in any other proportion. For having joined the like points of a different division in another set of vertical lines, transfer the divisions of the horizontal lines in the former figure to the corresponding equal lines in the new figure, and through these points draw curve lines which, in theory at least, will answer 
answer the same purpose as the straight lines radiating from $\mathbf{C}$ do in the other figure. The curve for refraction will be alike easily constructed in either figure; and the curve for parallax may always be laid down by help of a line of sines. In the same way it is easy to see how the vertical lines in the second diagram of the method described in the preceding part of this paper, admit of being divided in any proportion; as also how a portion of the triangle $\mathrm{ABC}$ in that figure might be expanded or contracted withont using square roots of the sines. I am, gentlemen,

Your most obedient servant, Henky Mexkie.

P.S.-It has frequently been remarked, that the accuracy of altitudes taken near the horizon is not to be trusted on account of the uncertainty of the refraction; and a similar objection though on a different ground has been raised against altitudes taken near the zenith. These remarks I do not mean to controvert; but it is no less true, that there are some cases of frequent occurrence, in which such altitudes although uncertain in themselves produce little or no uncertainty in clearing the lunar distance. When the distance is in a vertical circle, any error or nncertainty in altitude is apt to occasion a like error in distance. As the distance however deviates from the vertical position, the effect of an error in altitude will generally diminish; and when both altitudes are small and nearly equal, it would require a great error in altitude to affect the distance materially. Equal altitudes of any magnitude may thus be used without much danger of error. But it might easily be shown that, when both objects are in the horizon, the effect either of uncertainty in refraction, or of refraction itself, is not sensible on the distance: hence the observed horizontal distance if near to $90^{n}$ may be safely used as the true; because the effect of parallax then vanishes likewise; and since the effect of parallax on the horizontal distance, when that differs from a quadrant, may be so readily had from the 13th of the Requisite Tables, every opportunity should be embraced of measuring the lunar distance when both objects are in the horizon.

The foregoing graphical-methods are scarcely suited to very small altitudes; but still they may approximate to the truth when the altitudes are small and nearly equal, if the effect of refraction which they give be divided by the number of degrees +1 contained in both altitudes; then the quotient being subtracted from the above effect will leave the true effect of refraction pretty nearly. 
In taking an altitude very near the zenith for clearing the distance, it is sufficient if we can only be sure of its being within two degrees of the zenith, provided the lower altitude and the distance (not less than $20^{\circ}$ ) be properly observed; for if to the lower altitude we add the distance, the sum or its supplement may for ordinary purposes be used instead of the real apparent altitude of the higher body; and the reduction of the distance in such cases, will be the sum or difference of the corrections in altitude. But I do not mean to say that this obviates the effects of the spheroidal figure of the earth; nor have I endeavoured to determine how far the effects of small uncertainties in altitude might be obviated; at present I only meant to bring some cases which appeared to be neglected into more general notice.

\section{On some new Tables of Aberration and Nutation. By} Francis Baily, Esq. F.R.S.

\section{To the Editors of the Philosophical Magazine and Journal.}

Gentlemen, - $\mathbf{H}_{\text {aving }}$ been engaged, a short time since, with my friend Mr. Benjamin Gompertz, in investigating the principles of Aberration and Nutation, with a view to the formation of more correct tables on the subject, it occurred to us that an improvement might be made in the arrangement of the arguments of the usual tables for finding these quantities.

If we denote the obliquity of the ecliptic, the right ascension and declination of the star, the true longitude of the sun, the true longitude of the moon, and the mean longitude of the moon's node, by $\omega, R, D, \odot, D$ and $\&$ respectively, we shal! have the following correct and general formulæ for determining the above-mentioned quantities.

\section{Right Ascension.}

1. $-20^{\prime \prime}, 2550(\cos \omega \cdot \cos A \cdot \cos \odot+\sin A \cdot \sin \odot) \sec \mathrm{D}$

2. $\left\{\begin{array}{l}-1^{\prime \prime}, 2255 \sin 2 \odot \\ -\left(0^{\prime \prime}, 5799 \cos \mathbb{R} . \cos \right.\end{array}\right.$

3. $\left\{-15^{\prime \prime}, 3958 \sin 8\right.$

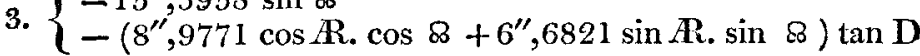

4. $+0^{\prime \prime}, 0877 \cos$ A. $\cos 28 \cdot \tan D$

5. $\left\{\begin{array}{l}-0^{\prime \prime}, 1846 \sin 2 D \\ -\left(0^{\prime \prime}, 0874 \cos A \cdot \cos 2 D+\cdot 0801 \sin R \cdot \sin 2 D\right) \tan D .\end{array}\right.$ 